\title{
VARIATION OF THE BRANCH POINTS FOR AN ANALYTIC FUNCTION
}

\author{
BY
}

A. W. GOODMAN

1. Introduction. Let

$$
w=f(z)=\sum_{n=1}^{\infty} a_{n} z^{n}, \quad a_{1}>0,
$$

be regular in $|z|<1$, and map that domain onto a Riemann surface $R$, having at least one simple branch point at $w=B=f(C)$. Consequently $f^{\prime}(C)=0$ and $f^{\prime \prime}(C) \neq 0$, and $0<|C|<1$. We form a new Riemann surface $R^{*}$, from $R$, by moving the branch point at $B$ to $B^{*}=B+\lambda$, where $\lambda$ is a sufficiently small complex number, while holding the boundary and all other branch points of $R$ fixed. Clearly $R^{*}$ is simply-connected and hence there is a uniquely determined regular function

$$
f^{*}(z)=\sum_{n=1}^{\infty} a_{n}^{*} z^{n}, \quad a_{1}^{*}>0,
$$

mapping $|z|<1$ onto $R^{*}$, and taking $z=0$, into the same place $w=0$ on $R$, as $f(z)$ does.

Our objective is to find a formula for $f^{*}(z)$ in terms of $f(z), \lambda$, and $\bar{\lambda}$ with error $O\left(\lambda^{2}\right)$. In fact we will prove that under the conditions just stated

$$
\begin{aligned}
f^{*}(z)=f(z)-z f^{\prime}(z) & \left\{\frac{\lambda z}{C^{2} f^{\prime \prime}(C)(C-z)}\right. \\
& \left.\quad+\operatorname{con}\left(\frac{\lambda}{C^{2} f^{\prime \prime}(C)}\right) \frac{\bar{C} z}{1-\bar{C} z}+\Re \frac{\lambda}{C^{2} f^{\prime \prime}(C)}\right\}+O\left(\lambda^{2}\right) \\
=f(z)-\frac{z f^{\prime}(z)}{2}\left\{\frac{\lambda}{C^{2} f^{\prime \prime}(C)} \frac{C+z}{C-z}\right. & \left.\quad+\operatorname{con}\left(\frac{\lambda}{C^{2} f^{\prime \prime}(C)}\right) \frac{1+\bar{C} z}{1-\bar{C} z}\right\}+O\left(\lambda^{2}\right),
\end{aligned}
$$

(where $\operatorname{con}($ ) denotes the conjugate of the quantity enclosed by the parentheses).

Formula (1.3) promises to be quite helpful in settling extremal problems for classes of functions in which the competing functions have branch points.

Presented to the Society December 28, 1956; received by the editors November 10, 1956. 
One rather surprising application to the theory of univalent functions will appear in a subsequent paper.

It would be desirable to have a formula similar to (1.3) in the case that $B$ a branch point of order $k$ is moved to $B^{*}$, or still better when a branch point of order $k$ is replaced by a number of neighboring branch points of total order $k$. Our method does not seem to be suitable for this latter problem, and even in the simpler case where $B$ and $B^{*}$ are both of order $k$, the computations become extremely involved. In what follows we will first assume that $B$ and $B^{*}$ are both of order $k$, but at an appropriate place in the proof we will consider only the cases $k=1$ and $k=2$. Our method is similar to that used by Schiffer [2], where the boundary of $R$ and all the branch points were subjected to a small variation.

2. Proof of the variational formula. Let $F(w)$ and $F^{*}(w)$ be the inverse functions of $f(z)$ and $f^{*}(z)$ respectively, mapping $R$ and $R^{*}$ onto $|z|<1$, and let $G(w, 0)=-\log |F(w)|$ and $G^{*}(w, 0)=-\log \left|F^{*}(w)\right|$ be the Green's functions for $R$ and $R^{*}$ with respect to the place 0 . Let $G(w, t)$ be the Green's function for $R$ with respect to the place $t$ on $R$. Our first aim is to compute

$$
K(w, 0)=G^{*}(w, 0)-G(w, 0)
$$

for $w \in \tilde{R}$, a certain Riemann surface contained in both $R$ and $R^{*}$.

We form $\widetilde{R}$ by deleting from $R$ the $k+1$ sheeted circle $\Delta:|w-B| \leqq r$, i.e., those places $w$ lying on the $k+1$ sheets joined at $B$, for which the coordinates satisfy this inequality. Here $r$ is selected small enough so that $\Delta$ is contained in $R$. If $\Gamma$ is the boundary of $\Delta$, the surface $\widetilde{R}$ could be obtained from $R^{*}$ by deleting the closure of the $k+1$ sheeted region bounded by $\Gamma$ with branch point at $B^{*}$, providing only that $|\lambda|<r$. Thus both $G^{*}(w, 0)$ and $G(w, 0)$ are defined over $\widetilde{R}$.

If we apply Green's formula [1, p. 12] to $K(w, 0)$ which is harmonic in $R$ we have, for $w \in R$

$$
K(w, 0)=\frac{1}{2 \pi} \int\left[K(t, 0) \frac{\partial}{\partial n} G(t, w)-G(t, w) \frac{\partial}{\partial n} K(t, 0)\right] d s
$$

where $t$, the variable of integration, runs over the boundary of $\widetilde{R}$, and $n$ is the normal into the region $\widetilde{R}$. On the boundary of $R, K(t, 0)=G(t, w)=0$, so in Equation (2.2) it is sufficient to integrate over $\Gamma: t=B+r e^{i \phi}, 0 \leqq \phi$ $\leqq 2(k+1) \pi$.

If $w \in \widetilde{R}, G(w, t)$ is harmonic for $t$ in $\Delta$, and if $r$ is taken sufficiently small, the place 0 will lie outside $\Delta$, and $G(t, 0)$ will also be harmonic in $\Delta$; so that

$$
\int_{\Gamma}\left[G(t, 0) \frac{\partial}{\partial n} G(t, w)-G(t, w) \frac{\partial}{\partial n} G(t, 0)\right] d s=0 .
$$

If now, in Equation (2.1), $w$ is replaced by $t$, and the result substituted in 
Equation (2.2), one obtains

$$
K(w, 0)=\frac{1}{2 \pi} \int_{\Gamma}\left[G^{*}(t, 0) \frac{\partial}{\partial n} G(t, w)-G(l, w) \frac{\partial}{\partial n} G^{*}(t, 0)\right] d s,
$$

taking into account Equation (2.3).

To evaluate the integral it is convenient to introduce a uniformizing variable $v^{k+1}=q^{k+1} e^{i(k+1) \psi}=t-B=r e^{i \phi}$. Then in the integral $d s=r d \phi$ $=(k+1) q^{k+1} d \psi$ and

$$
\frac{\partial}{\partial n}=\frac{\partial}{\partial r}=\frac{1}{(k+1) q^{k}} \frac{\partial}{\partial q} .
$$

Let $F(t, w)$ be a function regular on $R$ and mapping $R$ in a 1-1 manner onto $|z|<1$, as a function of $t$ on $R$, taking the place $w$ into the origin, and let

$$
F(t, w)=\sum_{n=0}^{\infty} D_{n}(t-B)^{n /(k+1)}=\sum_{n=0}^{\infty} D_{n} v^{n}
$$

in a neighborhood of the branch point $B$. Further let

$$
\begin{aligned}
F^{*}(t) & =\sum_{n=0}^{\infty} C_{n}^{*}\left(t-B^{*}\right)^{n /(k+1)} \\
& =\sum_{n=0}^{\infty} C_{n}^{*}\left(v^{k+1}-\lambda\right)^{n /(k+1)}
\end{aligned}
$$

in a neighborhood of $B^{*}$. With these substitutions the integral (2.4) becomes

$$
\begin{aligned}
K(w, 0)=\frac{q}{2 \pi} \int_{0}^{2 \pi} & \left\{\log \left|\sum_{n=0}^{\infty} C_{n}^{*}\left(v^{k+1}-\lambda\right)^{n /(k+1)}\right| \frac{\partial}{\partial q} \log \left|\sum_{n=0}^{\infty} D_{n} v^{n}\right|\right. \\
& \left.-\log \left|\sum_{n=0}^{\infty} D_{n} v^{n}\right| \frac{\partial}{\partial q} \log \left|\sum_{n=0}^{\infty} C_{n}^{*}\left(v^{k+1}-\lambda\right)^{n /(k+1)}\right|\right\} d \psi .
\end{aligned}
$$

If in the integrand of Equation (2.7) the terms $\log S$ are replaced by $\log S \bar{S} / 2$, it then appears as though $K(w, 0)$ is analytic in the variables $\lambda$ and $\bar{\lambda}$. Strictly speaking this is not obvious, because the coefficients $C_{n}{ }^{*}$ depend on $\lambda$, and at this moment we are only assuming that $C_{n}^{*}$ is a continuous function of $\lambda$ for $n=0,1,2, \cdots$. However this difficulty is avoided by regarding the $C_{n}^{*}$ as constants, and asking for the formal development of the integrand in Equation (2.7) as a double power series in $\lambda$ and $\bar{\lambda}$. Thus we can write

$$
K(w, 0)=A_{00}+A_{10} \lambda+A_{01} \bar{\lambda}+O\left(\lambda^{2}\right),
$$

where $A_{i j}$ depends upon the coefficients $C_{n}{ }^{*}, D_{n}$, and $q$.

A tedious computation from Equation (2.7) yields 


$$
\begin{aligned}
A_{10}= & \left.\frac{\partial K(w, 0)}{\partial \lambda}\right|_{\lambda=\bar{\lambda}=0} \\
= & -\frac{1}{8(k+1) \pi} \int_{0}^{2 \pi}\left\{\frac{\sum_{n=0}^{\infty} \sum_{m=0}^{\infty} n C_{n}^{*} \bar{C}_{m}^{*} q^{n+m-k} e^{i(n-m-k-1) \psi}}{\sum_{n=0}^{\infty} \sum_{m=0}^{\infty} C_{n}^{*} \bar{C}_{m}^{*} q^{n+m} e^{i(n-m) \psi}}\right. \\
& \cdot \frac{\partial}{\partial \dot{q}} \log \sum_{n=0}^{\infty} \sum_{m=0}^{\infty} D_{n} \bar{D}_{m} q^{n+m} e^{i(n-m) \psi}-q \log \sum_{n=0}^{\infty} \sum_{m=0}^{\infty} D_{n} \bar{D}_{m} q^{n+m} e^{i(n-m) \psi} \\
& \left.\cdot \frac{\partial}{\partial q} \frac{\sum_{n=0}^{\infty} \sum_{m=0}^{\infty} n C_{n}^{*} \bar{C}_{m}^{*} q^{n+m-k-1} e^{i(n-m-k-1) \psi}}{\sum_{n=0}^{\infty} \sum_{m=0}^{\infty} C_{n}^{*} C_{m}^{*} q^{n+m} e^{i(n-m) \psi}}\right\} d \psi,
\end{aligned}
$$

with a similar expression for $A_{01}$, which we can avoid since $A_{01}=\bar{A}_{10}$.

Now, with $\lambda$ fixed, $K(w, 0)$ is independent of the radius $r$ of $\Gamma$, for $|\lambda|$ $<r<r_{1}$, so that $K(w, 0)$ is independent of $q$ for a certain interval. Hence if the series (2.8) were rearranged in a Laurent series in terms of $q$, all of the coefficients would vanish except for the constant term. This means that in Equation (2.9) we may disregard all terms in the product which contain $q^{n}, n \neq 0$. An easy computation now gives $A_{00}=0$,

$$
\begin{aligned}
& A_{10}=-\frac{1}{4} \frac{C_{1}^{*} D_{1}}{C_{0}^{*} D_{0}}, \quad \text { if } k=1, \\
& A_{10}=\frac{1}{6} \frac{C_{1}^{*} D_{1}}{C_{0}^{*} D_{0}}\left(\frac{C_{1}^{*}}{C_{0}^{*}}+\frac{D_{1}}{D_{0}}\right)-\frac{1}{3} \frac{C_{1}^{*} D_{2}+C_{2}^{*} D_{1}}{C_{0}^{*} D_{0}} \quad \text { if } k=2,
\end{aligned}
$$

and still more complicated expression for larger $k$. Henceforth we consider only the cases $k=1,2$.

The next task is to determine the coefficients $D_{n}$ in terms of the mapping function $F(w)=F(w, 0)$ which maps $R$ onto $|z|<1$, with the place 0 on $R$ going into $z=0$. If $F(t, 0)=\tau$, then it is easy to see that

$$
F(w, t)=A \frac{F(w, 0)-\tau}{1-\bar{\tau} F(w, 0)}, \quad|A|=1,
$$

is the function mapping $R$ onto $|z|<1$, taking the place $t$ on $R$ into $z=0$. Here $A$ is a constant, suitably chosen to make $F^{\prime}(t, t)>0$. If we interchange $w$ and $t$, and replace $F(t, 0)=\tau$ by $F(w, 0)=z$, then

$$
F(t, w)=A \frac{F(t, 0)-z}{1-\bar{z} F(t, 0)}
$$


defines the same function, as the series (2.5).

Now let

$$
F(w, 0)=\sum_{n=0}^{\infty} C_{n}(w-B)^{n / k+1}, \quad C_{0}=C,
$$

in a neighborhood of the branch point $B$. Then substituting this development into Equation (2.13) and comparing coefficients with the series (2.5) yields:

$$
\begin{aligned}
\frac{D_{1}}{D_{0}} & =\frac{C_{1}\left(1-|z|^{2}\right)}{(C-z)(1-\bar{z} C)}, \\
\frac{D_{2}}{D_{0}} & =\frac{\left(C_{2}+\bar{z} C_{1}^{2}-\bar{z} C C_{2}\right)\left(1-|z|^{2}\right)}{(C-z)(1-\bar{z} C)^{2}} .
\end{aligned}
$$

We now have for $w \in \tilde{R}$, that

$$
K(w, 0)=\log \left|F(w) / F^{*}(w)\right|=2 \Re\left(A_{10} \lambda\right)+O\left(\lambda^{2}\right),
$$

where in the two cases $k=1,2, A_{10}$ is given by Equations (2.10) and (2.11) together with the Equations (2.15) and (2.16). If $\Phi(z)$ is an analytic function of $z$ such that $\Re \Phi(z)=2 \Re\left(A_{10} \lambda\right)$ and if $A^{*}$ is a suitable real constant, determined by the conditions $F^{\prime}(0)>0, F^{* \prime}(0)>0$, then for $w \in \tilde{R}$,

$$
\log F(w) / F^{*}(w)=\Phi(z)+i A^{*}+O\left(\lambda^{2}\right) .
$$

As will be seen presently both $\Phi(z)$ and $i A^{*}$ are $O(\lambda)$, so that Equation (2.17) yields

$$
\begin{aligned}
F^{*}(w) & =F(w) e^{-\left(\Phi(z)+i A^{*}+O\left(\lambda^{2}\right)\right)} \\
& =F(w)\left[1-\Phi(z)-i A^{*}+O\left(\lambda^{2}\right)\right] .
\end{aligned}
$$

From Equation (2.19) it is clear that for the coefficients of $F^{*}(w)$ we have $C_{n}^{*}=C_{n}+O(\lambda)$, and therefore in Equations (2.10) and (2.11) we may replace $C_{n}^{*}$ by $C_{n}$ without altering the order of the error term in Equation (2.17).

We now return to the $z$-plane and expand

$$
f(z)=w=f^{*}\left(F^{*}(w)\right)=f^{*}(F(w)+d)
$$

as a Taylor series about $F(w)=z$, where $z$ lies in $E^{\prime}=F(\tilde{R}) \cap F^{*}(\tilde{R})$, and $d=-F(w)\left(\Phi(z)+i A^{*}\right)+O\left(\lambda^{2}\right)$. We find easily that

$$
\begin{aligned}
f(z) & =f^{*}(F(w))+d f^{* \prime}(F(w))+O\left(d^{2}\right) \\
& =f^{*}(z)+d f^{* \prime}(z)+O\left(\lambda^{2}\right) .
\end{aligned}
$$

Since $f^{* \prime}(z)=f^{\prime}(z)+O(\lambda)$, we have for $z \in E^{\prime}$

$$
f^{*}(z)=f(z)+z f^{\prime}(z)\left(\Phi(z)+i A^{*}\right)+O\left(\lambda^{2}\right) .
$$

To determine $\Phi(z)$, first in the case $k=1$, we have from Equations (2.10) and (2.15) that 


$$
\begin{aligned}
2 \Re\left(A_{10} \lambda\right) & =\frac{-1}{2} \Re\left\{\frac{C_{1}^{*} C_{1}\left(1-|z|^{2}\right) \lambda}{C_{0}^{*}(C-z)(1-\bar{z} C)}\right\} \\
& =-\frac{1}{2} \Re\left\{\frac{C_{1}^{*} C_{1} \lambda}{C_{0}^{*}(C-z)}+\frac{C_{1}^{*} C_{1} \bar{z} \lambda}{C_{0}^{*}(1-\bar{z} C)}\right\} .
\end{aligned}
$$

If the last term in the braces is replaced by its conjugate, the real part is not altered, but the term becomes analytic in $z$. We can select for $\Phi(z)$, the function

$$
\Phi(z)=-\frac{1}{2}\left\{\frac{C_{1}^{*} C_{1} \lambda}{C_{0}^{*}(C-z)}+\operatorname{con}\left(\frac{C_{1}^{*} C_{1} \lambda}{C_{0}^{*}}\right) \frac{z}{1-\bar{C}_{z}}\right\} .
$$

The constant $A^{*}$ is determined from Equation (2.18) by the condition that $\Phi(0)+i A^{*}$ is real. This gives $A^{*}=\Im C_{1}^{*} C_{1} \lambda / 2 C C_{0}^{*}$.

It is now clear that $\Phi(z)$ and $A^{*}$ are $O(\lambda)$, as was announced, so that in Equation (2.22) the superscripts * may be dropped. Finally, from Equation (2.14), with $k=1$, and the fact that $F(w)$ is the inverse function to $f(z)$, we find that $C_{1}^{2}=2 / f^{\prime \prime}(C)$. Using this, and the modified form of $\Phi(z)$, together with $i A^{*}=-\Phi(0)+\Re \Phi(0)$, in Equation (2.21) will give Equation (1.3), valid for $z$ in $E^{\prime}$.

To conclude the proof of the theorem stated in the introduction, we must show that Equation (1.3) is valid throughout the unit circle. This is quite simple, because for sufficiently small $\lambda, E^{\prime}$ contains some ring $r^{\prime}<|z|<1$. But the term $O(\lambda)$ on the right side of Equation (1.3) is analytic in $z$, since the pole at $z=C$ inside the braces, is removed by the zero of $f^{\prime}(z)$ at $C$, and $f^{\prime \prime}(C)$ $\neq 0$. Thus any bound valid in the ring, is likewise valid, by the maximum modulus principle, throughout the unit circle.

In the case $k=2$, a long calculation, using Equations (2.15) and (2.16) in Equation (2.11) yields

$$
A_{10}=\frac{1}{6}\left\{\frac{C_{1}^{3}(2 C-z)}{C^{2}(C-z)^{2}}-\frac{4 C_{1} C_{2}}{C(C-z)}+\frac{C_{1}^{3} \bar{z}(1-2 \bar{z} C)}{C^{2}(1-\bar{z} C)^{2}}-\frac{4 C_{1} C_{2} \bar{z}}{C(1-\bar{z} C)}\right\},
$$

where the superscript ${ }^{*}$ has been dropped. Therefore we can take for our $\Phi(z)$, the function

$$
\begin{aligned}
\Phi(z)=\frac{1}{3}\left\{\frac{C_{1}^{3}(2 C-z) \lambda}{C^{2}(C-z)^{2}}-\frac{4 C_{1} C_{2} \lambda}{C(C-z)}+\operatorname{con}\left(\frac{C_{1}^{3} \lambda}{C^{2}}\right) \frac{z(1-2 \bar{C} z)}{(1-\bar{C} z)^{2}}\right. \\
\left.-4 \operatorname{con}\left(\frac{C_{1} C_{2} \lambda}{C}\right) \frac{z}{1-\bar{C} z}\right\}
\end{aligned}
$$

which is analytic in $z$, and for which $\Re \Phi(z)=2 \Re\left(A_{10} \lambda\right)$. In this case, 


$$
A^{*}=-\Im \Phi(0)=\frac{-1}{3} \Im\left(\frac{2 C_{1}^{3} \lambda}{C^{3}}-\frac{4 C_{1} C_{2} \lambda}{C^{2}}\right) .
$$

From $\Phi(z)+i A^{*}=\Phi(z)-\Phi(0)+\Re \Phi(0)$, we find

$$
\begin{aligned}
\Phi(z)+i A^{*}= & \frac{1}{3}\left\{\frac{C_{1}^{3} \lambda\left(3 C_{z}-2 z^{2}\right)}{C^{3}(C-z)^{2}}-\frac{4 C_{1} C_{2} \lambda z}{C^{2}(C-z)}\right. \\
& +\operatorname{con}\left(\frac{C_{1}^{3} \lambda}{C^{2}}\right) \frac{z-2 \bar{C} z^{2}}{(1-\bar{C} z)^{2}}-4 \operatorname{con}\left(\frac{C_{1} C_{2} \lambda}{C}\right) \frac{z}{1-\bar{C}_{z}} \\
& \left.+\Re\left(\frac{2 C_{1}^{3} \lambda}{C^{3}}-\frac{4 C_{1} C_{2} \lambda}{C^{2}}\right)\right\}
\end{aligned}
$$

If, in Equation (2.14), the coefficients $C_{1}, C_{2}$ are computed in terms of the inverse function, with $k=2$, one finds easily,

$$
C_{1}^{3}=6 / f^{\prime \prime \prime}(C), 4 C_{1} C_{2}=-2 f^{\mathrm{Iv}}(C) / f^{\prime \prime \prime}(C)^{2} .
$$

Using these results in Equation (2.26) and substituting in Equation (2.21) yields the formula:

$$
\begin{aligned}
f^{*}(z)= & f(z)+z f^{\prime}(z)\left\{\frac{2 \lambda\left(3 C z-2 z^{2}\right.}{C^{3} f^{\prime \prime \prime}(C)(C-z)^{2}}+\frac{2 f^{\mathrm{IV}}(C) \lambda z}{3\left(C f^{\prime \prime \prime}(C)\right)^{2}(C-z)}\right. \\
& +\operatorname{con}\left(\frac{2 \lambda}{C^{3} f^{\prime \prime \prime}(C)}\right) \frac{\bar{C} z-2 \bar{C}^{2} z^{2}}{(1-\bar{C} z)^{2}}+\frac{2}{3} \operatorname{con}\left(\frac{f^{\mathrm{IV}}(C) \lambda}{\left(C f^{\prime \prime \prime}(C)\right)^{2}}\right) \frac{C \bar{z}}{1-\bar{C} z} \\
& \left.+\Re\left(\frac{4 \lambda}{C^{3} f^{\prime \prime \prime}(C)}+\frac{2 f^{\mathrm{IV}}(C) \lambda}{3\left(C f^{\prime \prime \prime}(C)\right)^{2}}\right)\right\}+O\left(\lambda^{2}\right)
\end{aligned}
$$

This gives the variation of the mapping function $f(z)$ when a branch point of order 2 at $f(C)$ is moved a small amount $\lambda$.

3. An example function. Let $R$ be the surface consisting of the doublycovered circle $|w|<1$, with the branch point at $B=b e^{i \beta}, b>0$. By using a sequence of elementary functions it is easy to show that

$$
f(z)=\frac{2 b^{1 / 2} e^{i \beta} z-(1+b) z^{2}}{(1+b) e^{i \beta}-2 b^{1 / 2} z}
$$

maps $|z|<1$ onto $R$ with $f(0)=0, f^{\prime}(0)>0$. Now let $f^{*}(z)$ be the function (3.1) when $b e^{i \beta}$ is replaced by $B^{*}=b^{*} e^{i \beta *}=B+\lambda$, where $|\lambda|<\min \{b, 1-b\}$, and set $\delta f(z)=f^{*}(z)-f(z)$. Then direct computation, using Equation (3.1) will give 
(3.2) $\delta f(z)=z e^{i \beta} \frac{1-b}{b^{2}}\left\{\frac{\left(C-\bar{C} z^{2}\right) \Re\left(\lambda e^{-i \beta}\right)+(1-b) z i \Im\left(\lambda e^{-i \beta}\right)}{\left(C+\bar{C}^{-1}-2 z\right)^{2}}\right\}+O\left(\lambda^{2}\right)$

where $C=b^{1 / 2} e^{i \beta}$ is the critical point for the function (3.1). On the other hand if the formula (1.3) is applied to the function (3.1), one also obtains the variation (3.2).

\section{REFERENCES}

1. Z. Nehari, Conformal mapping, New York, 1952.

2. M. Schiffer, 'Variation of the Green function and theory of the p-valued functions, Amer. J. Math. vol. 65 (1943) pp. 341-360.

The Institute for Advanced Study And

THE UNIVERSITY OF KENTUCKY 